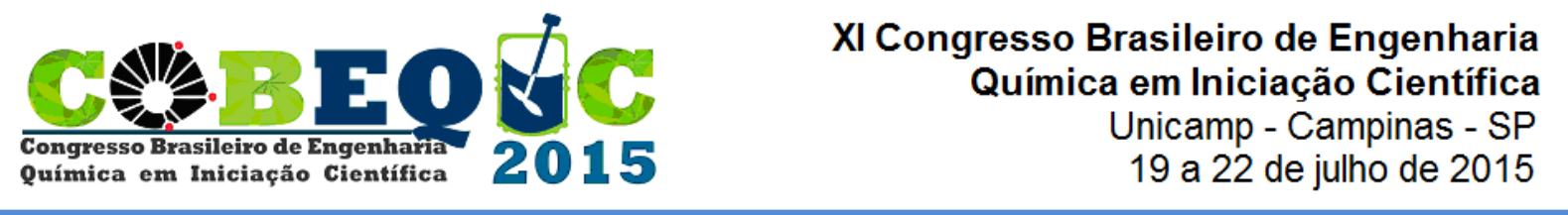

\title{
PROGRAMA JAVA PARA ESTIMATIVAS DE PROPRIEDADES TERMOFÍSICAS POR CONTRIBUIÇÃO DE GRUPOS FUNCIONAIS
}

\author{
L. G. Oliveira, J. C. Rocha, S. P. Nascimento, J. A. Cavalcante, N. A. Costa \\ Universidade Federal da Paraíba, Departamento de Engenharia Química \\ E-mail para contato: nagelalves@hotmail.com
}

\begin{abstract}
RESUMO - O presente trabalho tem por objetivo apresentar um programa JAVA para estimativas de propriedades termofísicas (propriedades críticas, temperatura normal de ebulição, temperatura de fusão, capacidade calorífica a pressão constante, entalpia de formação e energia livre de Gibbs de formação) usando grupos funcionais. $\mathrm{O}$ projeto do programa foi dividido em quatro partes: a) banco de dados contendo os parâmetros do modelo de contribuição de grupos funcionais, b) criação de caixas de diálogos para interação do usuário com o programa, c) codificações de subprogramas JAVA para as estimativas das propriedades termofísicas e d) validação dos resultados fornecidos pelo programa. O resultado final é um aplicativo, para fins educacionais e/ou comerciais, capaz de fornecer estimativas das propriedades termofísicas das espécies por contribuição de grupos funcionais com rapidez e confiabilidade.
\end{abstract}

\section{INTRODUÇÃO}

Os cálculos dos processos químicos, análises e projetos requerem propriedades características de uma substância pura, tais como: propriedades críticas; temperatura normal de ebulição; temperatura de fusão e propriedades de gases ideais (capacidade calorífica e propriedades de formação). Em vários casos, estes dados não são disponíveis, tornando-se necessárias predições dessas propriedades. Nos métodos de contribuições de grupos funcionais, as propriedades de uma substância são consideradas como sendo a soma das contribuições individuais de cada grupo estrutural, tais como: $-\mathrm{CH}_{3},>\mathrm{CH}_{2}$, OH e outros que, quando adicionados, formam a molécula.

Atualmente várias propriedades são preditas através dos métodos de contribuição de grupos funcionais: coeficiente de atividade; propriedades críticas; pressão de vapor; entalpia de vaporização; propriedades dos gases ideais; viscosidade e tensão superficial.

JAVA é uma linguagem de programação orientada a objetos, de alto nível que permite o rápido desenvolvimento de aplicações com interface gráfica. Sua escolha se deve à disponibilidade para as principais plataformas do mercado (Windows, Mac OS X, Linux) e facilidade de desenvolvimento. Após a compilação de um aplicativo JAVA é gerado um executável que pode ser executado em qualquer plataforma com suporte a linguagem JAVA e seus toolkits. Essa característica é possível pelo fato de que a aplicação JAVA é executada sobre uma máquina virtual, a JVM (Java Virtual Machine). 


\subsection{Contribuição de Grupos}

Reid et al. (1987), Poling et al. (2001) e Tester e Modell (1997) descrevem as equações e os parâmetros desenvolvidos por Joback e modificados por Lyndersen para a contribuição dos grupos funcionais que predizem propriedades termofísicas descritas abaixo.

Temperatura crítica: $T_{c}=T_{b}\left[0,584+0,965 \sum_{j} v_{j} \Delta_{T j}-\left(\sum_{j} v_{j} \Delta_{T j}\right)^{2}\right]^{-1}$, onde $T_{c}$ em K e $T_{b}$ é a temperatura normal de ebulição.

Pressão crítica: $\quad P_{c}=\left(0,113+0,0032 n_{A}-\sum_{j} v_{j} \Delta_{P j}\right)^{-2}$, onde $P_{c}$ em bar e $n_{A}$ é o número de átomos da molécula.

Volume crítico: $V_{c}=17,5+\sum_{j} v_{j} \Delta_{V j}$, onde $V_{c} \mathrm{em} \mathrm{cm}^{3} / \mathrm{mol}$;

Temperatura normal de ebulição: $T_{b}=178+\sum_{j} v_{j} \Delta_{T b j}$, onde $T_{b}$ em Kelvin

Temperatura de fusão: $T_{f}=122+\sum_{j} v_{j} \Delta_{T f j}$, onde $T_{f}$ em Kelvin;

Energia livre de Gibbs de formação: $\left.\Delta G_{f}^{\circ}\right|_{298,15 \mathrm{~K}}=53,88+\sum_{j} v_{j} \Delta_{G j}$, onde $\left.\Delta G_{f}^{\circ}\right|_{298,15 \mathrm{~K}} \mathrm{em}$ $\mathrm{kJ} / \mathrm{mol}$

Entalpia de formação: $\left.\Delta H_{f}^{\circ}\right|_{298,15 \mathrm{~K}}=68,29+\sum_{j} n_{j} \Delta_{H j}$, onde $\left.\Delta H_{f}^{\circ}\right|_{298,15 \mathrm{~K}} \mathrm{em} \mathrm{kJ} / \mathrm{mol}$;

Capacidade calorífica a pressão constante: $C_{P}^{0}=A+B T+C T^{2}+D T^{3}$, onde $C_{P}^{\circ}$ em J/mol.K. Os coeficientes da Equação (8) são obtidos por contribuição de grupos funcionais através das seguintes relações:

Coeficientes: $\quad A=\sum_{j} v_{j} \Delta_{a j}-37,93 ; \quad B=\sum_{j} v_{j} \Delta_{b j}+0,210 ; \quad C=\sum_{j} n_{j} \Delta_{c j}-3,91 \times 10^{-4} \quad \mathrm{e}$ $D=\sum_{j} n_{j} \Delta_{d j}+2,06 \times 10^{-7}$, onde $v_{j}$ é a quantidade de grupos funcionais $j$ na molécula.

As constantes características dos grupos funcionais $\Delta_{T j}, \Delta_{P j}, \Delta_{V j}, \Delta_{T b j}, \Delta_{T f j}, \Delta_{G j}, \Delta_{H j}, \Delta_{a f}$, $\Delta_{b f}, \Delta_{c f}$ e $\Delta_{d f}$ são fornecidas por Reid et al. (1987) e Poling et al. (2001).

\section{METODOLOGIA}


A metodologia adotada para o desenvolvimento dos subprogramas JAVA é esquematizada na Figura 1.

Figura 1 - Contribuição de Grupos Funcionais

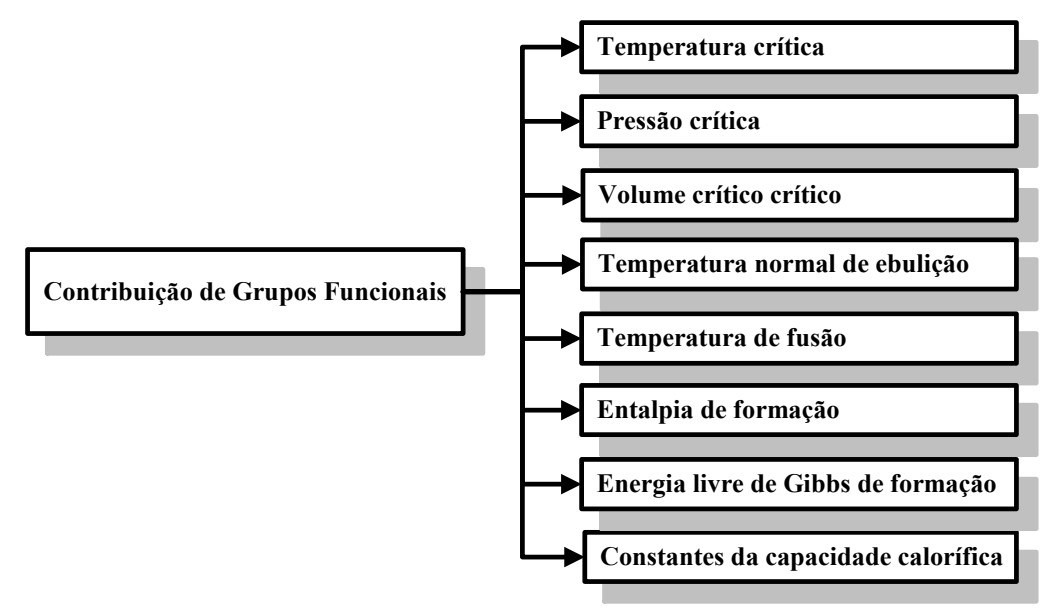

\subsection{Esquema do desenvolvimento do aplicativo}

Na Figura 2 é mostrada a janela principal do aplicativo desenvolvido em Java.

Figura 2 - Janela principal do aplicativo Java 


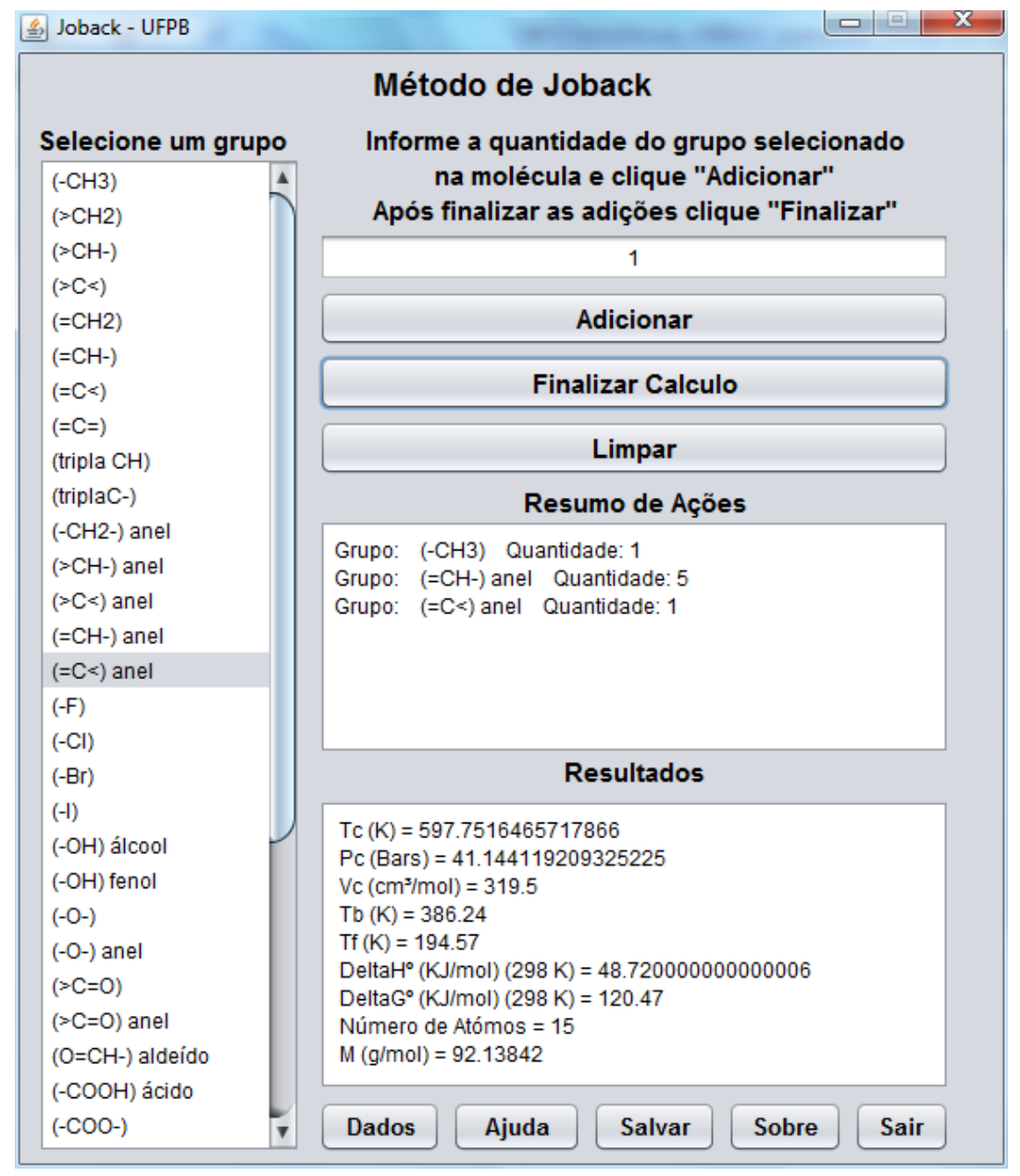

\subsection{Descrições das opções da janela principal}

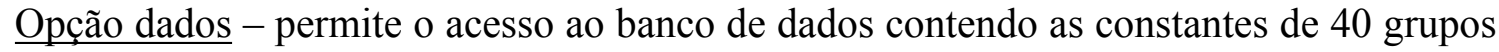
funcionais do método de Joback reportadas por Poling et al. (1987) (Figura 3).

Opção Salvar: abre a caixa de diálogo mostrada na Figura 4 que permite ao usuário especificar o local e o nome do arquivo de saída de dados.

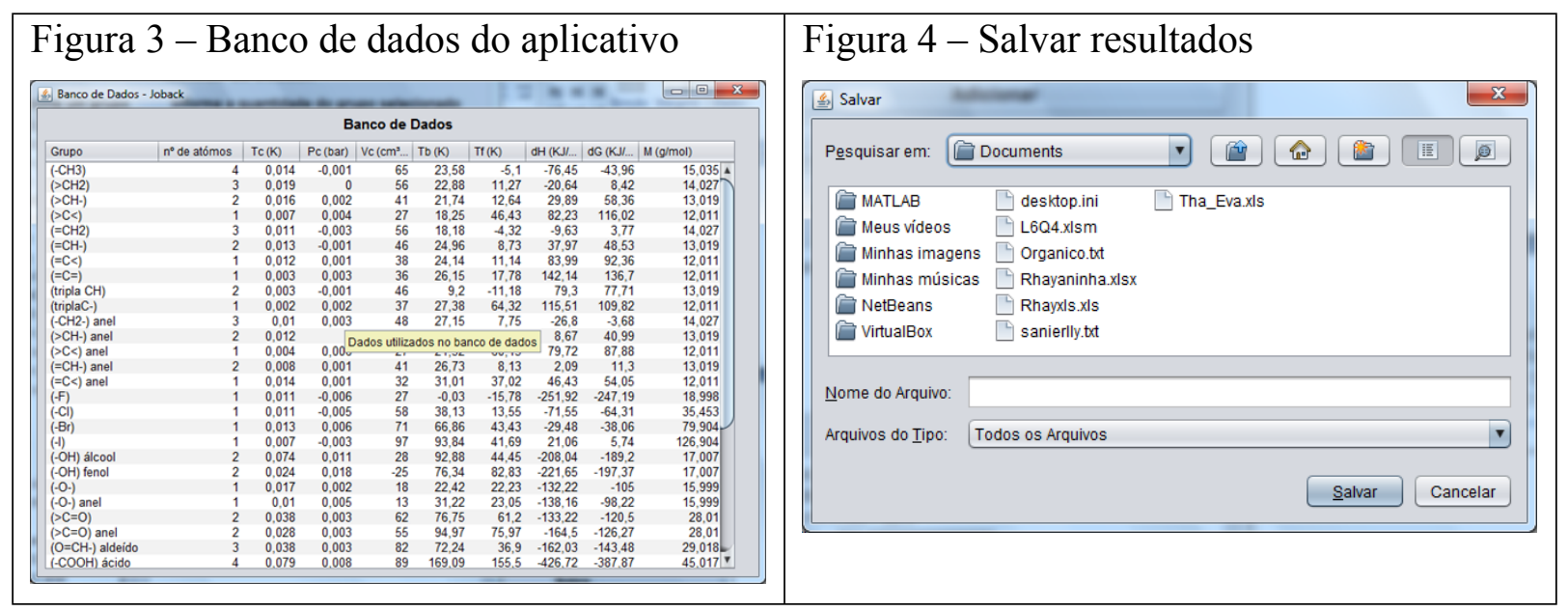


Opção Ajuda: descrição resumida do modelo de contribuição de grupos funcionais de Joback (Figura 5).

Opção Sobre: informações sobre os autores do aplicativo (Figura 6).

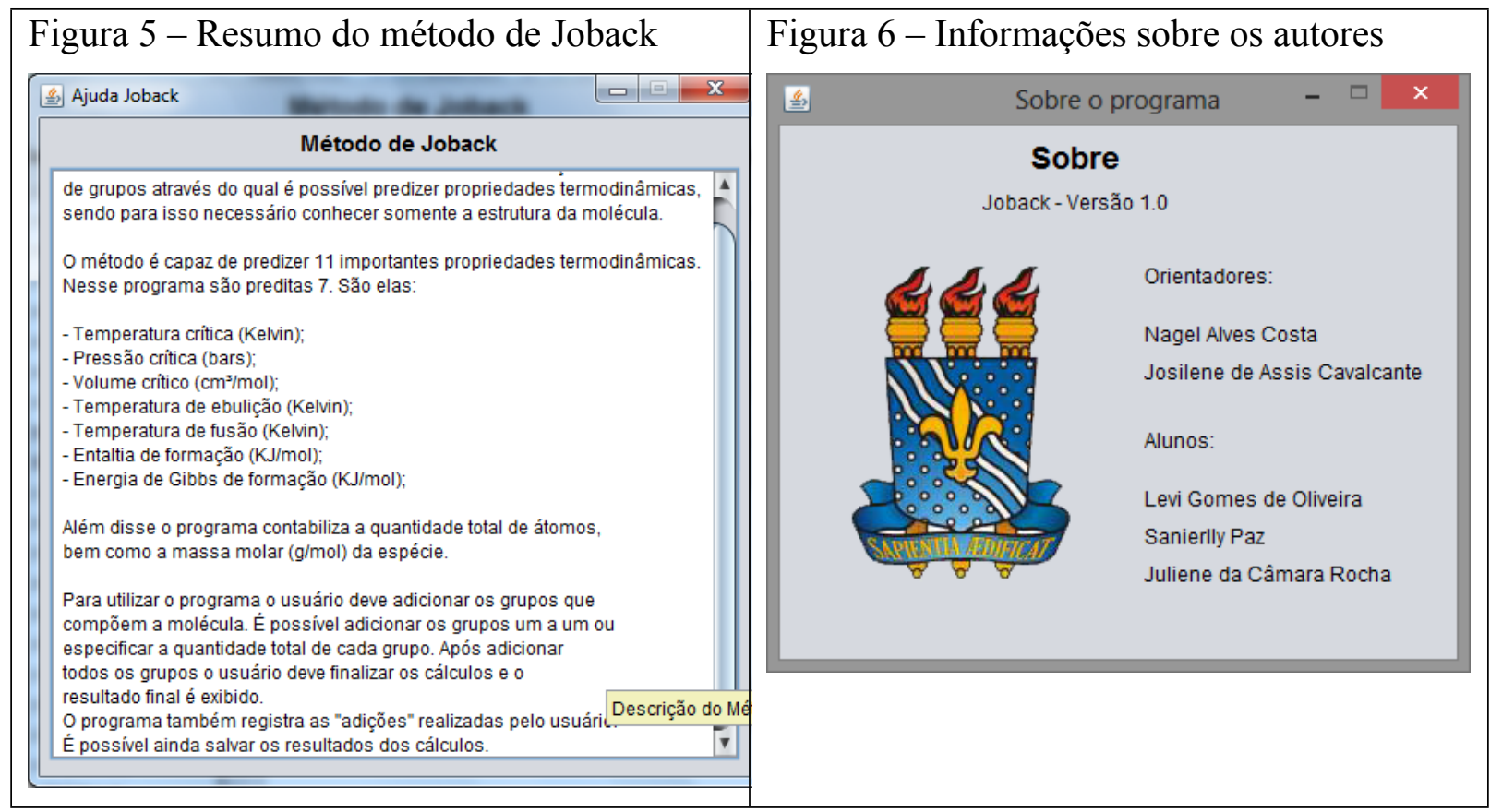

Opção Sair: fecha o aplicativo (Figura 2).

Lista dos grupos funcionais: apresenta a lista de grupos funcionais do método de Joback (Figura 2).

Caixa de texto: permite especificar a quantidade de cada grupo escolhido (Figura 2).

Opção adicionar: permite ao usuário adicionar os grupos funcionais para configurar a fórmula estrutura da espécie química (Figura 2).

Opção finalizar cálculo: finaliza os cálculos (Figura 2).

Opção limpar: limpa a saída de dados e remove os locais de memória especificados pelo usuário (Figura 2).

\section{VALIDAÇÃO DO APLICATIVO}

A validação do aplicativo Java desenvolvido para as predições das propriedades termofísicas por contribuição de grupos funcionais foi realizada através da comparação dos valores reportados na literatura mundial com os fornecidos pelo aplicativo desenvolvido. Os valores da literatura (em negrito) foram reportados por: Tester e Modell (1997) para o tolueno e diclorometano e Poling et al. (2001) para o 2-etilfenol.

Nas Tabelas 1, 2 e 3 são apresentadas, respectivamente, as propriedades críticas e a temperatura normal de ebulição, as propriedades termodinâmicas dos gases ideais e as constantes da capacidade calorífica a pressão constante de gases ideais. 
Tabela 1 - Propriedades críticas

\begin{tabular}{lcccc}
\hline Espécie & $T_{b}(\mathrm{~K})$ & $T_{c}(\mathrm{~K})$ & $P_{c}(\mathrm{bar})$ & $V_{c}\left(\mathrm{~cm}^{3} / \mathrm{mol}\right)$ \\
\hline \multirow{2}{*}{ Tolueno } & $\mathbf{3 8 6 , 2 4}$ & $\mathbf{5 9 7 , 7}$ & $\mathbf{4 1 , 1}$ & $\mathbf{3 1 9 , 5}$ \\
& 386,24 & 597,75 & 41,14 & 319,5 \\
\hline \multirow{2}{*}{ Diclorometano } & $\mathbf{2 9 7 , 1 4}$ & $\mathbf{4 7 8 , 5 5}$ & $\mathbf{5 1 , 9 1}$ & $\mathbf{1 8 9 , 5}$ \\
& 297,14 & 478,55 & 51,906 & 189,5 \\
\hline \multirow{2}{*}{ 2-etilfenol } & $\mathbf{4 8 9 , 7 4}$ & $\mathbf{7 1 5 , 7}$ & $\mathbf{4 4 , 0 9}$ & $\mathbf{3 4 1 , 5}$ \\
& 489,74 & 715,75 & 44,091 & 341,5 \\
\hline
\end{tabular}

Tabela 2 - Propriedades termodinâmicas do gás ideal no estado padrão de 298,15 K

\begin{tabular}{lcc|cc}
\hline \multirow{2}{*}{ Espécie } & \multicolumn{2}{c|}{ Literatura $(\mathrm{kJ} / \mathrm{mol})$} & \multicolumn{2}{c}{ Aplicativo $(\mathrm{kJ} / \mathrm{mol})$} \\
\cline { 2 - 5 } & $\Delta H_{f}^{\circ}$ & $\Delta G_{f}^{\circ}$ & $\Delta H_{f}^{\circ}$ & $\Delta G_{f}^{\circ}$ \\
\hline Tolueno & $\mathbf{4 8 , 7 2}$ & $\mathbf{1 2 0 , 4 7}$ & 48,72 & 120,47 \\
Diclorometano & $\mathbf{- 9 5 , 4 5}$ & $\mathbf{- 6 6 , 3 2}$ & $-95,45$ & $-66,32$ \\
2-etilfenol & $\mathbf{- 1 4 9 , 2 3}$ & $\mathbf{- 2 5 , 7 3}$ & $-149,23$ & $-25,73$ \\
\hline
\end{tabular}

Tabela $3-\mathrm{C}_{\mathrm{P}}=A+B T+C T^{2}+D T^{3}$

\begin{tabular}{lcccc}
\hline Espécie & $A$ & $B$ & $C \times 10^{4}$ & $D \times 10^{8}$ \\
\hline \multirow{2}{*}{ Tolueno } & $\mathbf{- 3 7 , 3 8}$ & $\mathbf{0 , 5 8 9 9}$ & $\mathbf{- 3 , 8 8}$ & $\mathbf{9 , 8 0}$ \\
& $-37,38$ & 0,58992 & $-3,882$ & 9,76 \\
\hline \multirow{2}{*}{ Diclorometano } & $\mathbf{- 6 2 , 2 3}$ & $\mathbf{0 , 1 1 2 4}$ & $-\mathbf{7 , 1 0}$ & $\mathbf{1 , 9 0}$ \\
& 27,761 & 0,1124 & $-7,14$ & 1,87 \\
\hline \multirow{2}{*}{ 2-etilfenol } & $\mathbf{- 4 7 , 2 0 9}$ & $\mathbf{0 , 8 3 9 5 2}$ & $\mathbf{- 6 , 9 8 4}$ & $\mathbf{2 , 4 1 9}$ \\
& $-47,209$ & 0,83952 & $-6,9896$ & 2,426 \\
\hline
\end{tabular}

Foram verificadas as excelentes concordâncias entre os resultados fornecidos pelo aplicativo com os valores reportados na literatura. As pequenas diferenças observadas nos cálculos mostrados ao longo do trabalho são devido às diferenças entre as precisões numéricas utilizadas pelos pesquisadores e pelo aplicativo.

Na Tabela 3 ocorreu uma discordância no coeficiente $A$ do diclorometano. Tester e Modell (1997) reporta que o grupo (-CH2-) possui um valor $\Delta_{a}=-9,09 \times 10^{-1}$, o que esta de acordo com REID et al. (1988) e POLING et al. (2001). Entretanto, no cálculo do coeficiente $A$, os pesquisadores utilizaram $\Delta_{a}=-9,09 \times 10^{+1}$.

\section{CONCLUSÃO}

Este trabalho apresenta um aplicativo capaz de fornecer com confiabilidade e rapidez as estimativas de propriedades termofísicas usando a metodologia de contribuição de grupos funcionais. Deve ser observado que a confiabilidade da estimativa de uma determinada propriedade termofísica está associada à efíciência do modelo fornecido pelo pesquisador e não por erros numéricos ou erros de codificações dos subprogramas VBA.

\section{REFERÊNCIAS}


POLING, B. E.;PRAUSNITZ, J. M.; O’CONNELL, J. P. The properties of gases and liquids. McGraw-Hill, Fifth Edition, 2001

REID, R. C., PRAUSNITZ, J. M., POLING, B. E. The properties of gases and liquids. McGraw-Hill, Fourth Edition, 1987.

TESTER, J. W., MODELL, M. Thermodynamics and its Applications. Prentice Hall, 3rd Edition, 1997 